\title{
Performance Analysis of Single and Multiuser MIMO Diversity Channels Using Nakagami- $m$ Distribution
}

\author{
Kai-Kit Wong, Member, IEEE
}

\begin{abstract}
This letter studies the diversity advantage of single and multiuser systems utilizing multiple-input multiple-output (MIMO) antennas for known channels at the transmitter and receiver(s) by investigating the overall distribution of the resulting channel. For the single-user case, the resultant channel is taken as the largest singular value of the MIMO channel whereas for the multiuser scenario, the resultant channel is obtained by a recently proposed joint-channel diagonalization. The channel distribution is studied using hypothesis testing with the null hypothesis that the distribution follows a Nakagami- $m$ distribution with given parameters. It is concluded from the chi-square goodness-of-fit test that the distribution of the resultant channel for single or multiuser MIMO channel is well matched with a Nakagami- $m$ distribution.
\end{abstract}

Index Terms-Diversity, joint-channel diagonalization (JCD), multiple-input multiple-output (MIMO), Nakagami distribution.

\section{INTRODUCTION}

$\mathbf{T}$ O PROFIT from the ever-growing demand for highquality high-data-rate wireless communications, powerand-spectral efficient techniques are sought. Utilizing multiple antennas at both transmitter and receiver [known as multiple-input multiple-output (MIMO) antenna] is definitely one of the most outstanding candidates. In recent years, many advanced MIMO antenna systems have been demonstrated to provide excellent performance for both single [1]-[4] and multiuser [5], [6]-[8] communications.

In [1] and [2], space-time coding or Bell-Labs layered spacetime (BLAST), which does not require prior channel information at the transmitter, was studied. To exploit the full diversity of MIMO antennas, it is, however, advantageous for the transmitter to have prior knowledge of the channel. In [3] and [4], adaptation of antenna weights based on singular value decomposition (SVD) of known random channels was proposed.

Utilizing multiple antennas at the transmitter and all mobile receivers (downlink multiuser MIMO) for performance enhancement has also been considered (e.g., [4]-[8]). In [6] and [7], signal-to-interference plus noise ratio (SINR) enhancement using multielement transmit antenna array was proposed while later in [5], an algorithm was devised for decomposing multiuser MIMO channel matrices into multiple uncoupled single-user systems [i.e., orthogonal space division multiplexing (OSDM)]. More recently, Choi and Murch [8]

Manuscript received October 18, 2002; revised January 28, 2003, March 24, 2003; accepted March 30, 2003. The editor coordinating the review of this paper and approving it for publication is Z. Tian. This work was supported in part by the Hong Kong Research Grant Council and in part by the Hongkong Telecom Institute of Information Technology.

The author is with the Department of Electrical and Electronic Engineering,

The University of Hong Kong, Hong Kong (e-mail: kitwong@ieee.org).

Digital Object Identifier 10.1109/TWC.2004.830850 presented a null space projection method as a transmit preprocessing to decouple multiuser signals.

Motivated by the success of MIMO antenna systems, the focus of this letter is to study the diversity advantage using MIMO antennas by determining the probability density function (PDF) of the resultant channel for single and multiuser MIMO communications, through which the statistical characteristics of general MIMO systems can be completely described. For the single-user case, the resultant channel is taken as the largest singular value of the MIMO channel whereas for multiuser scenario, the resultant channel is obtained by the joint-channel diagonalization (JCD) of the multiuser MIMO channels [5]. For convenience, our consideration of multiuser MIMO will be limited to an $N_{t}$-element base station (BS) communicating with $N\left(=N_{t}\right)$ mobile stations (MS) each with two antenna elements.

The letter is organized as follows. In Section II, we review the single-user single-input multiple-output (SIMO) and MIMO systems. Section III presents the OSDM solution we use on multiuser MIMO channels. In Section IV, simulation setup and results are presented. We conclude this letter in Section V.

\section{SINGLE-USER SIMO AND MIMO SYSTEMS}

\section{A. SIMO With Maximal Ratio Combining Diversity}

For a single-user SIMO system where $N_{r}$ antennas are located at the receiver, the equivalent baseband received signal can be conveniently expressed in vector form as

$$
\mathbf{y}=\mathbf{h} z+\mathbf{n}
$$

where $\mathbf{y}=\left[y_{1} y_{2}, \ldots, y_{N_{r}}\right]^{T}$ with $y_{\ell}$ denoting the received signal at the $\ell$ th antenna and the superscript $T$ denoting the transpose operation. Vectors $\mathbf{h}$ and $\mathbf{n}$ are defined similarly and they represent, respectively, the complex fading coefficients of the radio channel and the zero-mean complex additive white Gaussian noise (CAWGN) with variance of $N_{0} / 2$ per dimension. Likewise, the scalar $z$ denotes the complex modulated symbol.

Optimal reception can be achieved by maximal ratio combining (MRC) so that the array output signal $\hat{z}$ is $\hat{z}=\mathbf{h}^{\dagger} \mathbf{y}$ where the superscript $\dagger$ denotes the conjugate transposition.

Denoting the resultant channel coefficient after MRC as $\beta \triangleq$ $\|\mathbf{h}\|$, it is well known [9]-[11] that an $N_{r}$-branch MRC (with uncorrelated fading) provides an $N_{r}$-fold diversity in terms of both receiving power (i.e., the channel gain)

$$
\Omega=E\left[\beta^{2}\right]
$$


and the inverse of the normalized variance of the squared channel gain (i.e., the diversity order [11])

$$
m=\frac{\Omega^{2}}{E\left[\left(\beta^{2}-\Omega\right)^{2}\right]} .
$$

In other words, for an $N_{r}$-branch MRC, $\Omega=N_{r} \Omega_{0}$ and $m=N_{r} m_{0}$ where $\Omega_{0}$ and $m_{0}$ are the diversity statistics of a single-user system without antenna diversity. When $m \rightarrow \infty$, channel becomes CAWGN.

Given that the fading coefficients $h_{\ell}$ are zero-mean complex Gaussian distributed, the PDF of $\beta$ has been found to be a Nakagami- $m$ distribution [9]-[11] and is given by [12]

$$
\operatorname{PDF}(\beta)=\frac{2 m^{m}}{\Gamma(m) \Omega^{m}} \beta^{2 m-1} \exp \left(-\frac{m \beta^{2}}{\Omega}\right)
$$

where $\Omega$ and $m$ are defined in (2) and (3), respectively, and $\Gamma(\cdot)$ denotes the gamma function.

\section{B. MIMO With SVD Diversity}

When $N_{t}$ antennas are employed at the transmitter side, the symbol $z$ before transmission will be multiplied by a complex transmit weight vector $\mathbf{t}$. The signal at each receive antenna is a noisy superposition of the $N_{t}$ transmitted signals perturbed by fading. This can be written as

$$
\mathbf{y}=\mathbf{H t} z+\mathbf{n}
$$

where $\mathbf{H} \in \mathbb{C}^{N_{r} \times N_{t}}$ is the MIMO channel matrix.

To detect the signal, an array output signal (similar to the case for SIMO system) is produced by $\hat{z}=\mathbf{r}^{\dagger} \mathbf{y}$ where $\mathbf{r}$ is the receive antenna vector. As found in [4], SVD can be used as the optimal antenna processing. Therefore, the transmit and receive weight vectors can be chosen, respectively, as the right and left singular vectors which correspond to the largest singular value of $\mathbf{H}$. The resulting system is then reduced to a single-input single-output (SISO) system with resultant channel determined by the channel largest singular value, $\sigma_{1}$. Hence, the resultant channel coefficient for a single-user MIMO with SVD diversity is $\beta=\sigma_{1}$.

In [4] and [13], it was found that for a $\left(N_{t}, N_{r}\right)=(2,2)$ system, the PDF of $\beta$ is given by

$\operatorname{PDF}(\beta)=2 \beta e^{-\beta^{2}}\left(\beta^{4}-2 \beta^{2}+2\right)-4 \beta e^{-2 \beta^{2}}, \quad$ for $\beta \geq 0$.

However, the PDF expression for other values of $N_{t}$ or $N_{r}$ is unavailable. Inspired by the idea of Nakagami- $m$ distribution for SIMO systems, we would like to re-express (6) in the form of Nakagami- $m$ distribution. In this case, when $\left(N_{t}, N_{r}\right)=(2,2)$, it can be shown that $(m, \Omega) \doteq(3.9,3.5)$. Substituting these values into (4), we obtain the following PDF:

$$
\operatorname{PDF}(\beta) \approx 0.5881 \beta^{6.7346} e^{-1.11 \beta^{2}}, \quad \text { for } \beta \geq 0 .
$$

Though (6) and (7) look very different, they are, in fact, extremely close with inappreciable difference (see Fig. 1).

The idea to express the PDF of $\beta$ using (4) provides an easy way for the procurement of the PDF of $\beta$ for larger values of $N_{t}$ or $N_{r}$ since the PDF can then be completely determined by the two statistics, $m$ and $\Omega$. The details will be discussed in the simulation section (see Section IV).

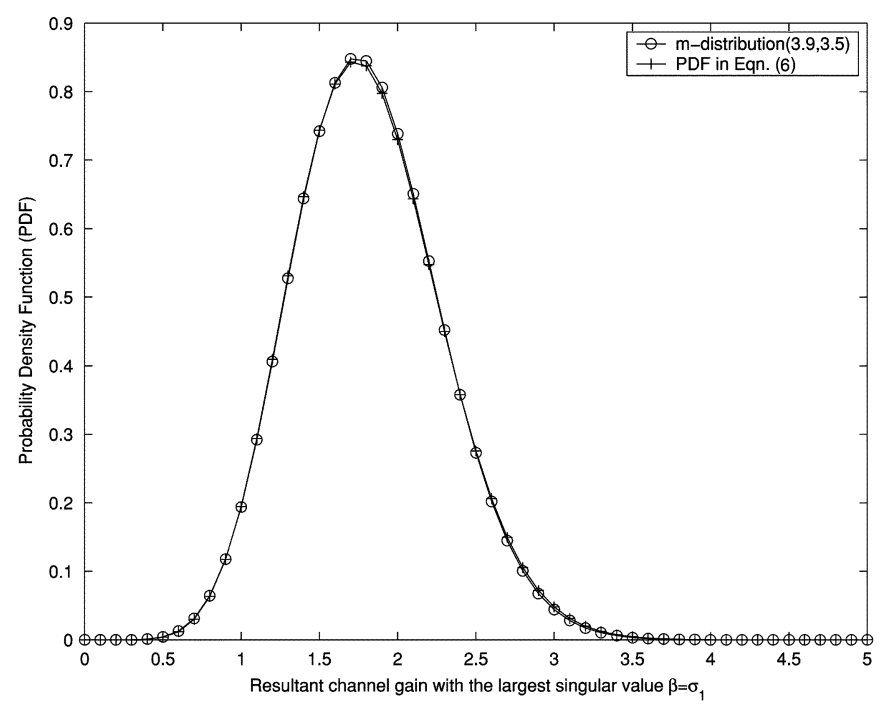

Fig. 1. Comparison for PDFs of Nakagami- $m$ distribution with $(m, \Omega)=$ $(3.9,3.5)$ and the expression in (6).

\section{MULTIUSER MIMO With JCD Diversity}

Consider a multiuser system where an $N$-element BS communicates simultaneously with $N$ 2-element mobile receivers. Using the system model (5), as described in Section II-B, we can write the multiuser MIMO system as [5], [7]

$$
\hat{z}_{n}=\mathbf{r}_{n}^{\dagger}\left(\sum_{k=1}^{N} \mathbf{H}_{n} \mathbf{t}_{k} z_{k}+\mathbf{n}_{n}\right) \quad \forall n
$$

where $\mathbf{n}_{n} \in \mathbb{C}^{2}$ is the noise vector, $z_{k} \in \mathbb{C}$ denotes the symbols (with time index omitted) transmitted from the $k$ th user, $\mathbf{t}_{k} \in$ $\mathbb{C}^{N}$ is the antenna weight vector for transmitting the $k$ th user signal, $\mathbf{H}_{n} \in \mathbb{C}^{2 \times N}$ is the channel matrix from the BS to the $n$th MS, and $\mathbf{r}_{n} \in \mathbb{C}^{2}$ is the antenna weight vector for signal reception by the $n$th user.

In dealing with multiuser communications, it is advantageous to handle with users in an orthogonal manner, as in conventional systems such as time, frequency or code division multiplexing (T/F/CDM). Through joint adaptation of the multiple antennas at the BS and MS, the idea has been extended in spatial domain [5].

In this letter, we shall adopt the iterative algorithm proposed in [5]. Since the multiuser MIMO system using JCD can be reduced to multiple uncoupled single-user systems, the overall system performance will then be solely determined by the statistics of the resultant channel $\beta_{n}$. To see if Nakagami- $m$ distribution can be used to represent the PDF of the resultant channel coefficient, $\beta_{n}$, in multiuser MIMO systems, simulations were carried out and results in Fig. 2 indicate that the PDF of $\beta_{n}$ for a five-user MIMO antenna system is well matched to a Nakagami distribution with $(m, \Omega)=(4.1,3.4)$. A more rigorous analysis using Chi-square goodness-of-fit test will be discussed in the following section.

\section{Simulation Setup And Results}

In this section, we shall provide the simulation results of channel gain and diversity order for various configurations followed by a Chi-square goodness-of-fit test. Perfect channel 


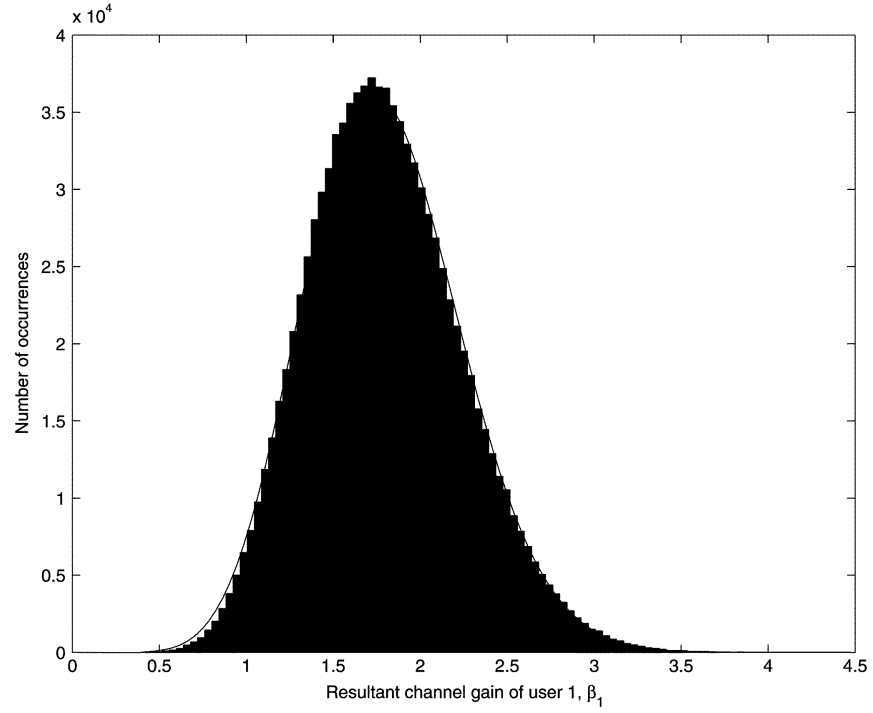

Fig. 2. Comparison between the theoretical values of Nakagami- $m$ distribution with $(m, \Omega)=(4.1,3.4)$ (solid line) and Monte Carlo simulations of a five-user MIMO using JCD diversity (strips).

state information is assumed and known to both the transmitter and receiver so that SVD or JCD can be performed. The radio channel is assumed to be in flat Rayleigh fading and the channels among transmit and receive antenna pairs are independent and identically distributed. For each simulation, $10^{4} \leq N_{s} \leq 10^{6}$ independent channel realizations are simulated and for each channel realization, the resultant channel gain(s), $\beta$ or $\beta_{n}$, is (are) computed.

\section{A. Diversity Analysis}

We measure the diversity advantage of a system in terms of $\Omega$ and $m$ [defined earlier in (2) and (3)] relative to a system without diversity. The benchmark system without diversity we use is a single-user system with a single transmit and receive antenna, where the diversity statistics $\Omega_{0}=m_{0}=1$.

Results in Table I provide the values of $\Omega$ and $m$ for single-user MIMO systems with various number of transmit and receive antennas. For any given number of transmit (or receive) antennas, the diversity statistics grow up linearly as the number of receive (or transmit) antennas. Therefore, we can use the following multiple linear regression model:

$$
\begin{aligned}
m_{\mathrm{SVD}}\left(N_{t, i}, N_{r, j}\right)=\beta_{t} N_{t, i}+\beta_{r} N_{r, j} & \\
& +\beta_{t, r} N_{t, i} N_{r, j}+\beta_{0}
\end{aligned}
$$

where $\beta_{t}, \beta_{r}, \beta_{t, r}$, and $\beta_{0}$ are the regression coefficients, $N_{t, i}=$ $i$, and $N_{r, j}=j$ for $i=2, \ldots, 5$, and $2 \leq j \leq i$. The regression coefficients are determined using least-squares criterion such that the sum of squared deviations between the observations and the regression surface is minimized. After solving this from our results, $m_{\mathrm{SVD}}$ can be conveniently approximated by

$m_{\mathrm{SVD}} \approx 0.9469 N_{t}+1.1961 N_{r}+0.3698 N_{t} N_{r}-1.9746$

with the sum of squared error equals 0.1344 . Similarly, we have also

$\Omega_{\mathrm{SVD}} \approx 1.1907 N_{t}+1.4908 N_{r}+0.0787 N_{t} N_{r}-2.1797$
TABLE I

RESUlts OF THE CHI-SQUARE TESTS FOR SINGLE-USER MIMO SystemS

\begin{tabular}{c|c|c|c|c}
\hline$N_{t}$ & $N_{r}$ & $m$ & $\Omega$ & $\chi_{\text {statistic }}^{2}$ \\
\hline 2 & 2 & 3.8673 & 3.4840 & $5.9880^{\star}$ \\
\hline 3 & 2 & 5.4542 & 4.8915 & $6.3360^{\star}$ \\
\cline { 2 - 5 } & 3 & 7.7263 & 6.5092 & $3.2580^{\star}$ \\
\hline \multirow{4}{*}{4} & 2 & 7.1314 & 6.1872 & $7.3020^{\star}$ \\
\cline { 2 - 5 } & 3 & 9.8662 & 8.0616 & $14.3360^{\star}$ \\
\cline { 2 - 5 } & 4 & 12.5216 & 9.7758 & $11.8760^{\star}$ \\
\hline 5 & 2 & 8.6438 & 7.4672 & $4.1840^{\star}$ \\
\cline { 2 - 5 } & 3 & 12.0152 & 9.5100 & $17.0000^{\star}$ \\
\cline { 2 - 5 } & 4 & 15.0209 & 11.3860 & 23.5140 \\
\cline { 2 - 5 } & 5 & 17.9214 & 13.1835 & 19.2420 \\
\hline 6 & 2 & 10.4650 & 8.7255 & $16.3580^{\star}$ \\
\cline { 2 - 5 } & 3 & 14.1357 & 10.9511 & $8.7600^{\star}$ \\
\cline { 2 - 5 } & 4 & 17.3895 & 12.9186 & 19.9980 \\
\cline { 2 - 5 } & 5 & 20.8344 & 14.8464 & $9.5560^{\star}$ \\
\cline { 2 - 5 } & 6 & 24.0821 & 16.5881 & 20.7200 \\
\hline
\end{tabular}

with the sum of squared deviations equals 0.1049. Note from these equations that the number of receive antennas $\left(N_{r}\right)$ plays a more important role on diversity gain than the number of transmit antennas $\left(N_{t}\right)$. This comes to a similar conclusion as in [14] that a SIMO antenna system will always outperform a MISO (multiple transmit antennas and a single receive antenna) antenna system with fixed numbers of total antenna elements. For example, as we can see in Table I, $\left(N_{t}, N_{r}\right)=(4,4)$ obtains higher diversity advantages compared to $\left(N_{t}, N_{r}\right)=(5,3)$, which is also better than a $\left(N_{t}, N_{r}\right)=(6,2)$ system.

Similar results for multiuser MIMO using JCD diversity are provided in Table II. $\Omega_{n}$ and $m_{n}$ denote, respectively, the channel gain and diversity order of the resultant channel for user $n$. Results in Table II reveal that for a two-user MIMO system, it has about 2.4 diversity gain of receiving power $\Omega_{n}$ and 2.6 diversity gain for reducing the effect of fading $m_{n}$, compared with a single-user system without diversity. It is important to note that the diversity orders obtained can be more than the number of receive antennas at the MS, meaning that in addition to support of multiple users, the transmit antennas at the BS can provide diversity to the MS's. More diversity can be achieved for a larger value of $N=N_{t}$. Generally speaking, both $\Omega_{n}$ and $m_{n}$ will increase as the number of transmit antennas increases even the number of users increases correspondingly.

It should also be noted that for a particular $N$, all users perform similarly and have nearly the same diversity statistics (i.e., $\Omega_{1} \approx \ldots \approx \Omega_{N}$ and $m_{1} \approx \cdots \approx m_{N}$ ). This agrees with the fact that, on average, all users are processed in the same way. Thus, to describe the diversity performances of the system, we use

$$
\bar{\Omega} \triangleq \frac{1}{N} \sum_{n=1}^{N} \Omega_{n}
$$

and

$$
\bar{m} \triangleq \frac{1}{N} \sum_{n=1}^{N} m_{n}
$$

as the diversity statistics of a $N$-user MIMO system using JCD diversity. The results for $\bar{m}$ and $\bar{\Omega}$ against the number of users $N$ are illustrated in Fig. 3. Results in this figure indicate that the diversity advantages for both $\bar{m}$ and $\bar{\Omega}$ grow almost linearly 
TABLE II

REsults OF THE CHI-SQUARE TESTS FOR MUlTIUSER MIMO Systems

\begin{tabular}{|c|c|c|c|c|c|c|}
\hline$N$ & User $n$ & $m_{n}$ & $\Omega_{n}$ & $\chi_{\text {statistic }}^{2}$ & $\bar{m}$ & $\bar{\Omega}$ \\
\hline \multirow[t]{2}{*}{2} & 1 & 2.5946 & 2.3988 & $4.7740^{\star}$ & \multirow[t]{2}{*}{2.6001} & \multirow[t]{2}{*}{2.3958} \\
\hline & 2 & 2.6057 & 2.3928 & $1.4980^{\star}$ & & \\
\hline \multirow[t]{3}{*}{3} & 1 & 3.1072 & 2.7686 & 18.8320 & \multirow[t]{3}{*}{3.0762} & \multirow[t]{3}{*}{2.7578} \\
\hline & 2 & 3.1048 & 2.7613 & $9.1900^{\star}$ & & \\
\hline & 3 & 3.0167 & 2.7436 & 19.1120 & & \\
\hline \multirow[t]{4}{*}{4} & 1 & 3.6065 & 3.1206 & $14.2360^{\star}$ & \multirow[t]{4}{*}{3.6709} & \multirow[t]{4}{*}{3.1094} \\
\hline & 2 & 3.7361 & 3.1109 & $4.5160^{\star}$ & & \\
\hline & 3 & 3.6580 & 3.0966 & $13.6400^{\star}$ & & \\
\hline & 4 & 3.6831 & 3.1095 & $17.2460^{\star}$ & & \\
\hline \multirow[t]{5}{*}{5} & 1 & 4.1412 & 3.4526 & $16.37^{\star}$ & \multirow[t]{5}{*}{4.1413} & \multirow[t]{5}{*}{3.4226} \\
\hline & 2 & 4.1747 & 3.4162 & $9.4240^{\star}$ & & \\
\hline & 3 & 4.1078 & 3.4349 & $10.2820^{\star}$ & & \\
\hline & 4 & 4.1349 & 3.4169 & $11.6120^{\star}$ & & \\
\hline & 5 & 4.1480 & 3.3925 & $9.7300^{\star}$ & & \\
\hline \multirow[t]{6}{*}{6} & 1 & 4.4858 & 3.7079 & 31.004 & \multirow[t]{6}{*}{4.5771} & \multirow[t]{6}{*}{3.7230} \\
\hline & 2 & 4.6294 & 3.7456 & $11.3120^{\star}$ & & \\
\hline & 3 & 4.5033 & 3.7180 & $13.6760^{\star}$ & & \\
\hline & 4 & 4.7190 & 3.7212 & $15.8720^{\star}$ & & \\
\hline & 5 & 4.6308 & 3.7254 & $13.2760^{\star}$ & & \\
\hline & 6 & 4.4943 & 3.7197 & $16.6320^{\star}$ & & \\
\hline \multirow[t]{7}{*}{7} & 1 & 5.0400 & 4.0233 & $14.406^{\star}$ & \multirow[t]{7}{*}{5.0713} & \multirow[t]{7}{*}{4.0169} \\
\hline & 2 & 5.0974 & 4.0046 & 24.1000 & & \\
\hline & 3 & 4.9712 & 4.0124 & 22.3100 & & \\
\hline & 4 & 5.1266 & 4.0076 & $12.8060^{\star}$ & & \\
\hline & 5 & 5.1557 & 4.0108 & $21.678^{\star}$ & & \\
\hline & 6 & 4.9769 & 4.0194 & $13.3900^{\star}$ & & \\
\hline & 7 & 5.1312 & 4.0402 & $12.368^{\star}$ & & \\
\hline \multirow[t]{8}{*}{8} & 1 & 5.6589 & 4.2735 & $4.8700^{\star}$ & \multirow[t]{8}{*}{5.5680} & \multirow[t]{8}{*}{4.2884} \\
\hline & 2 & 5.5658 & 4.2853 & $13.3660^{\star}$ & & \\
\hline & 3 & 5.5075 & 4.2977 & $16.1020^{\star}$ & & \\
\hline & 4 & 5.4698 & 4.2800 & $13.642^{\star}$ & & \\
\hline & 5 & 5.6627 & 4.2939 & $7.8280^{\star}$ & & \\
\hline & 6 & 5.7046 & 4.2929 & $7.1060^{\star}$ & & \\
\hline & 7 & 5.3986 & 4.3092 & 33.8840 & & \\
\hline & 8 & 5.5762 & 4.2745 & $16.0740^{\star}$ & & \\
\hline \multirow[t]{9}{*}{9} & 1 & 5.7715 & 4.5675 & $17.264^{\star}$ & \multirow[t]{9}{*}{5.9704} & 4.5597 \\
\hline & 2 & 6.0568 & 4.5644 & $17.3440^{\star}$ & & \\
\hline & 3 & 6.0435 & 4.5588 & $18.27200^{\star}$ & & \\
\hline & 4 & 5.8665 & 4.5591 & 23.2800 & & \\
\hline & 5 & 5.9517 & 4.5744 & $16.9500^{\star}$ & & \\
\hline & 6 & 5.8837 & 4.5667 & $14.5160^{\star}$ & & \\
\hline & 7 & 6.1012 & 4.5578 & $9.9100^{\star}$ & & \\
\hline & 8 & 5.9052 & 4.5412 & $10.8280^{\star}$ & & \\
\hline & 9 & 6.1533 & 4.5473 & $6.3580^{\star}$ & & \\
\hline
\end{tabular}

with $N$ and the gain does not seem to diminish as $N$ keeps increasing. Results demonstrate that about $0.4844(\approx 0.5)$ diversity gain for $\bar{m}$ and $0.3074(\approx 0.3)$ diversity gain for $\bar{\Omega}$ can be benefited for all users from every additional accommodated user. This can be explained by recognizing that in support of additional users via space, JCD gains additional diversity by better use of multiple channels with increased degrees of freedom. Similar to single-user MIMO systems, we can also have approximate formulas for computing the diversity order and channel gain of multiuser MIMO systems. The formulas are given by

and

$$
m_{\mathrm{JCD}} \approx 0.4844 N+1.6703
$$

$$
\Omega_{\mathrm{JCD}} \approx 0.3074 N+1.8434 .
$$

However, it should be noted that they are only useful for a system with $N$-element transmit antennas to communicate with $N$ simultaneous users, each with two antenna elements.

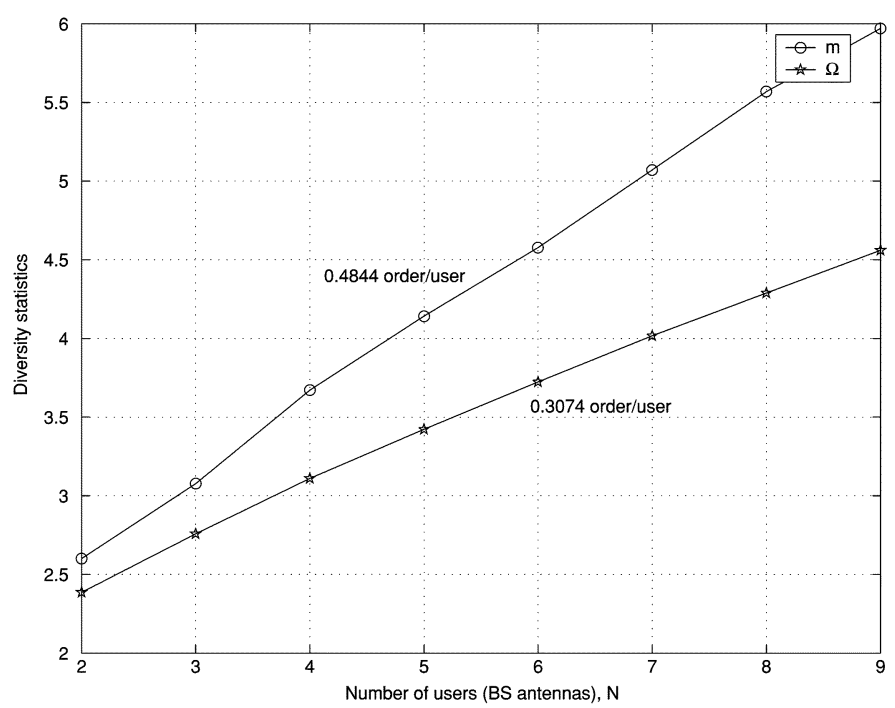

Fig. 3. Diversity gains of $N$-user MIMO systems using JCD diversity.

\section{B. Chi-Square Goodness-of-Fit Test}

In Fig. 2, we have seen that the histogram of simulated multiuser MIMO channels matches very well with the Nakagami- $m$ distribution with the same $m$ and $\Omega$. To demonstrate this more rigorously, we use the Chi-square goodness-of-fit test to check if the resultant channel coefficient for single or multiuser MIMO system is Nakagami- $m$ distributed, under significance level of $1 \%$.

To perform the test, we first calculate $\Omega$ and $m$ from the data according to (2) and (3) and the following hypothesis is set up:

$$
\begin{aligned}
& H_{0} \text { : follow a Nakagami distribution with }(m, \Omega) . \\
& H_{a} \text { : do not follow the given distribution. }
\end{aligned}
$$

Then, the data $\left(N_{s}\right.$ samples of $\beta$ ) are divided into ten bins and the test statistic $\chi_{\text {statistic }}^{2}$ is computed as

$$
\chi_{\text {statistic }}^{2}=\sum_{k=1}^{10} \frac{\left(O_{k}-E_{k}\right)^{2}}{E_{k}}
$$

where $O_{k}$ is the observed number of outcomes and $E_{k}$ is the expected number of outcomes that fall in the $k$ th interval. The expected frequency $E_{k}$ is calculated by

$$
E_{k}=N_{s}\left(F\left(U_{k}\right)-F\left(L_{k}\right)\right)
$$

where $U_{k}$ is the upper limit for class $k, L_{k}$ is the lower limit for class $k$, and $F(\cdot)$ is the cumulative distribution function (CDF) for the distribution, which is now given by [12]

$$
F(\beta)=\Gamma_{\text {inc }}\left(\frac{m \beta^{2}}{\Omega}, m\right)
$$

where $\Gamma_{\text {inc }}$ is the incomplete Gamma function. The test statistic, in principle, follows a Chi-square distribution with seven degrees of freedoms [15]. Let $\chi_{\mathrm{df}, 1-\alpha}^{2}$ be the Chi-square percent point function where $\mathrm{df}$ is the degrees of freedom and $\alpha$ is the significance level. In our case, $\mathrm{df}=7$ and we have set $\alpha=1 \%$. Therefore, $\chi_{\mathrm{df}, 1-\alpha}^{2}=\chi_{7,0.99}^{2}=18.48$. If $\chi_{\text {statistic }}^{2}<\chi_{\mathrm{df}, 1-\alpha}^{2}$, then we accept $H_{0}$; otherwise $H_{0}$ is rejected. 


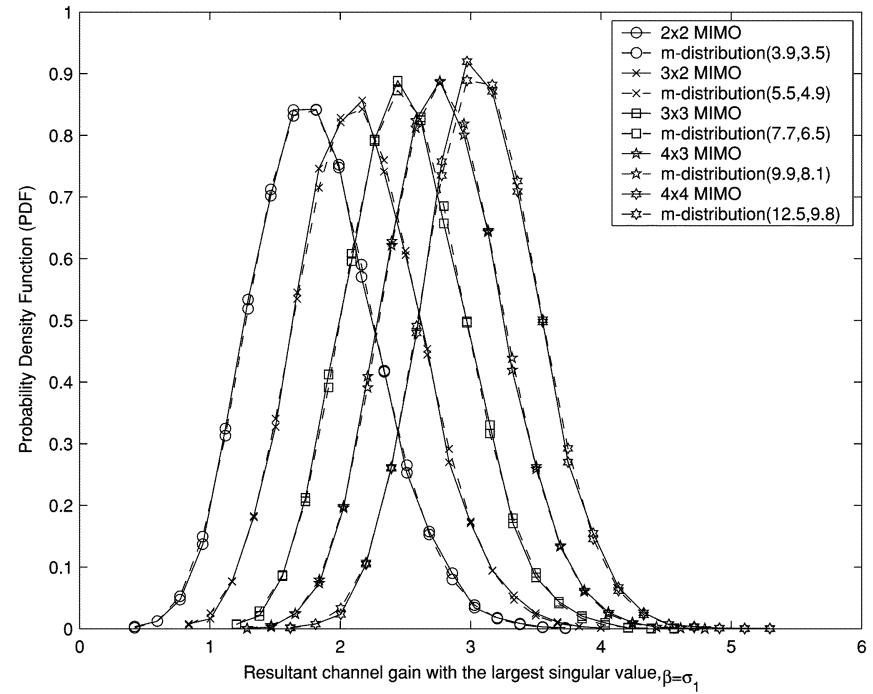

Fig. 4. PDFs of single-user MIMO systems using SVD diversity (solid lines) and Nakagami- $m$ distributions (dashed lines).

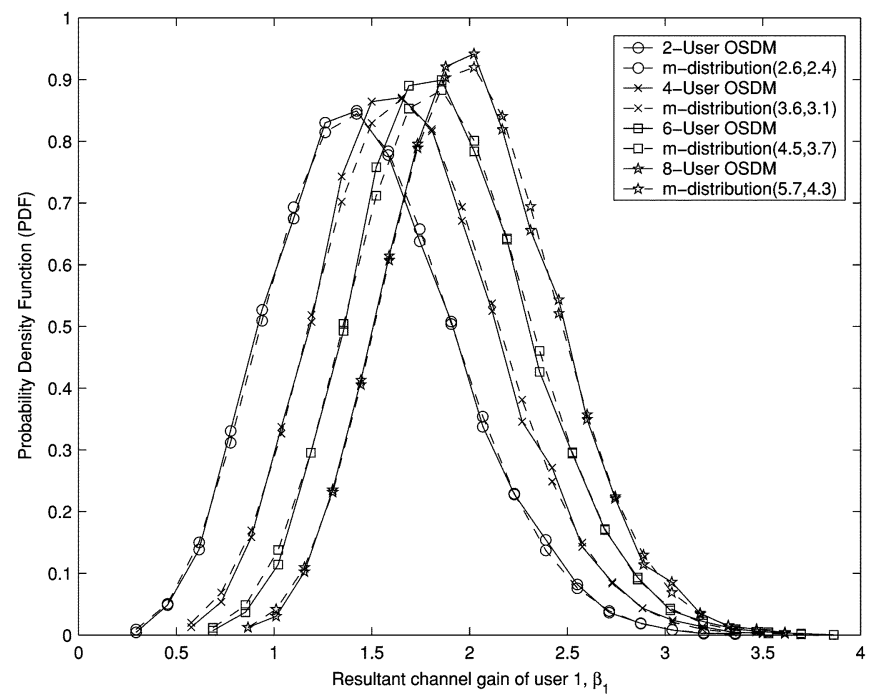

Fig. 5. PDFs of multiuser MIMO systems using JCD diversity (solid lines) and Nakagami- $m$ distributions (dashed lines).

The test statistics were calculated and listed in Tables I and II. The numbers marked with the superscript $\star$ indicate that the null hypothesis is accepted. Results in Table I show that 11 out of 15 configurations accept the null hypothesis. Similarly for multiuser channels, it is illustrated in Table II that 38 out of 44 configurations accept the null hypothesis. As a result, we can conclude that the PDF of single and multiuser MIMO channels can be well expressed (or approximated) as a Nakagami- $m$ distribution (4) with the diversity statistics that can be estimated by (10) and (11) for single-user MIMO or (14) and (15) for multiuser MIMO channels.

In Figs. 4 and 5, the PDFs of the simulated channels are compared to the PDF's of Nakagami- $m$ distribution with the same diversity statistics of the simulated channels. Results in Fig. 4 are provided for single-user MIMO channels using SVD diver- sity. As we can see, the PDFs of the simulated channels almost overlap with the PDFs of the Nakagami- $m$ distribution. The same is also true for multiuser MIMO channels concluded by the results in Fig. 5.

\section{CONClusion}

It is well understood that if prior channel knowledge is available at the transmitter, SVD or more generally JCD can be used to improve the system capacity and performance using MIMO channels. The focus of this letter is to quantify the diversity advantages achievable by the antennas and to investigate the overall distribution of the resultant channel of MIMO systems. Through Monte Carlo simulations, we end up with formulas to generally describe the channel gain and diversity order of single and multiuser MIMO systems. We have also concluded by Chisquare goodness-of-fit test that the distribution of the resultant channel gain can be well approximated by a Nakagami- $m$ distribution with corresponding $\Omega$ and $m$ for single and multiuser MIMO antenna systems.

\section{REFERENCES}

[1] V. Tarokh, N. Seshadri, and A. R. Calderbank, "Space-time codes for high data rate wireless communication: Performance criterion and code construction," IEEE Trans. Inform. Theory, vol. 44, pp. 744-765, Mar. 1998.

[2] G. J. Foschini, "Layered space-time architecture for wireless communication in a fading environment when using multi-element antennas," Bell Labs. Tech. J., vol. 1, no. 2, pp. 41-59, Aug. 1996.

[3] K. K. Wong, R. D. Murch, and K. B. Letaief, "Optimizing time and space MIMO antenna system for frequency selective fading channels," IEEE J. Select. Areas Commun., vol. 19, pp. 1395-1407, July 2001.

[4] J. B. Andersen, "Array gain and capacity for known random channels with multiple element arrays at both ends," IEEE J. Select. Areas Commun., vol. 18, pp. 2172-2178, Nov. 2000.

[5] K. K. Wong, R. D. Murch, and K. B. Letaief, "A joint channel diagonalization for multiuser MIMO antenna systems," IEEE Trans. Wireless Commun., vol. 2, pp. 773-786, July 2003.

[6] J. K. Cavers, "Single-user and multiuser adaptive maximal ratio transmission for Rayleigh channels," IEEE Trans. Veh. Technol., vol. 49, pp. 2043-2050, Nov. 2000.

[7] K. K. Wong, R. D. Murch, and K. B. Letaief, "Performance enhancement of multiuser MIMO wireless communications systems," IEEE Trans. Commun., vol. 50, pp. 1960-1970, Dec. 2002.

[8] R. L. U. Choi and R. D. Murch, "A transmit preprocessing technique for multiuser MIMO systems using a decomposition approach," IEEE Trans. Wireless Commun., vol. 3, pp. 20-24, Jan. 2004.

[9] N. Youssef, T. Munakata, and M. Takeda, "Fade statistics in Nakagami fading environments," in Proc. IEEE Int. Symp. Spread Spectrum Techniques and Applications, vol. 3, 1996, pp. 1244-1247.

[10] S. A. Abbas and A. U. Sheikh, "A geometric theory of Nakagami fading multipath mobile radio channel with physical interpretations," in Proc. IEEE Vehicular Technology Conf., vol. 2, 1996, pp. 637-641.

[11] M. S. Alouini and A. Goldsmith, "Capacity of Nakagami multipath fading channels," in Proc. IEEE Vehicular Technology Conf., vol. 1, 1997, pp. 358-362.

[12] M. Nakagami, "The $m$-distribution: A general formula of intensity distribution of rapid fading," in Statistical Methods in Radio Wave Propagation, W. C. Hoffman, Ed. New York: Pergamon, 1960, pp. 3-36.

[13] A. T. James, "Distribution of matrix variates and latent roots derived from normal samples," Ann. Math. Stat., vol. 35, pp. 475-501, 1964.

[14] M. Kang and M. S. Alouini, "Performance analysis of MIMO systems with co-channel interference over Rayleigh fading channels," in IEEE Int. Conf. Communications, vol. 1, New York, 2002, pp. 391-395.

[15] W. G. Cochran, "Some methods for strengthening the common chi-square tests," Biometrics 10, vol. 10, no. 4, pp. 417-451, 1954. 